\title{
The superfluid glass phase of ${ }^{3} \mathrm{He}-\mathrm{A}$
}

\author{
J. I. A. Li, J. Pollanen, A. M. Zimmerman, C. A. Collett, W. J. Gannon and W. P. Halperin^
}

It is established theoretically that an ordered state with continuous symmetry is inherently unstable to arbitrarily small amounts of disorder ${ }^{1,2}$. This principle is of central importance in a wide variety of condensed systems including superconducting vortices ${ }^{3,4}$, Ising spin models ${ }^{5}$ and their dynamics ${ }^{6}$, and liquid crystals in porous media 7,8 , where some degree of disorder is ubiquitous, although its experimental observation has been elusive. On the basis of these ideas, it was predicted ${ }^{9}$ that ${ }^{3} \mathrm{He}$ in high-porosity aerogel would become a superfluid glass. We report here our nuclear magnetic resonance measurements on ${ }^{3} \mathrm{He}$ in aerogel demonstrating destruction of long-range orientational order of the intrinsic superfluid orbital angular momentum, confirming the existence of a superfluid glass. In contrast, ${ }^{3} \mathrm{He}-\mathrm{A}$ generated by warming from superfluid ${ }^{3} \mathrm{He}-\mathrm{B}$ has perfect long-range orientational order providing a mechanism for switching off this effect.

Close to the absolute zero of temperature, liquid ${ }^{3} \mathrm{He}$ condenses into a $p$-wave superfluid of Cooper pairs resulting in two phases with fundamentally different symmetry: the isotropic B-phase and an anisotropic A-phase. In zero magnetic field, ${ }^{3} \mathrm{He}-\mathrm{A}$ appears in a small corner of the pressure versus temperature phase diagram shown in Fig. 1d. Its anisotropy, a paradigm for more recently discovered unconventional superconductors ${ }^{10}$, is characterized by the orientation of its order parameter defined by orbital angular momentum and spin induced by magnetic field, $\hat{\mathbf{l}}$ and $\hat{\mathbf{s}}$. The spin is necessarily aligned with an applied magnetic field, $\mathbf{H}$; however, the orbital angular momentum has continuous rotational symmetry. That symmetry can be broken, for example, at a wall or interface to which $\hat{\mathbf{I}}$ must be perpendicular, thereby defining a preferred direction on a macroscopic scale.

Volovik proposed ${ }^{9}$ that this long-range orientational coherence of angular momentum would be destroyed by random microscopic disorder that can be realized if the ${ }^{3} \mathrm{He}$ is imbibed in highly porous silica aerogel as shown in our simulation Fig. 1a,b. This sensitivity to small amounts of disorder on a microscopic scale was discussed by Larkin ${ }^{1}$ and Imry and $\mathrm{Ma}^{2}$ for a broad range of physical phenomena ${ }^{3-8}$ and we refer to this as the LIM effect. If this proposal is correct then in the LIM state the order parameter structure of the superfluid will be completely hidden, a behaviour of potential significance for understanding exotic superconductors such as $\mathrm{URu}_{2} \mathrm{Si}_{2}$ (ref. 11).

We use nuclear magnetic resonance (NMR) to look for the LIM state of superfluid ${ }^{3} \mathrm{He}-\mathrm{A}$, directly interrogating the orientation of $\hat{1}$ by measuring the Leggett shift ${ }^{12}$ of the NMR spectrum, $\Delta \omega_{\mathrm{A}}$. In pure ${ }^{3} \mathrm{He}$ this frequency shift is proportional to the nuclear dipole energy, $F_{\mathrm{D}} \propto-(\hat{\mathbf{l}} \cdot \hat{\mathbf{d}})^{2}$, where $\hat{\mathbf{d}}$ is a spin-space vector constrained to be perpendicular to $\hat{\mathbf{s}}$ while minimizing $F_{\mathrm{D}}$. This shift is strongly temperature dependent, but for an orbital glass it should be very small, or ideally zero (Supplementary Information) as we report here.
Leggett interpreted a shift in the NMR spectrum, centred at the frequency $\omega_{\mathrm{A}}(T)$, equation (1), as evidence of orientational order ${ }^{12}$,

$$
\omega_{\mathrm{A}}^{2}(T)=\omega_{\mathrm{L}}^{2}+\Omega_{\mathrm{A}}^{2}(T)
$$

in the absence of any external influences on $\hat{\mathbf{l}}$. A global minimum of dipole energy corresponds to $(\hat{\mathbf{l}}|| \hat{\mathbf{d}})$ giving a maximal frequency shift at large magnetic fields $(H>30 \mathrm{G})$,

$$
\Delta \omega_{\mathrm{A}} \equiv \omega_{\mathrm{A}}-\omega_{\mathrm{L}} \approx \Omega_{\mathrm{A}}^{2}(T) / 2 \omega_{\mathrm{L}}
$$

where $\omega_{\mathrm{L}}$ is the NMR frequency of the free atom. The longitudinal resonance frequency, $\Omega_{\mathrm{A}}$, is proportional to the amplitude of the maximum energy gap, going to zero at the transition temperature, $T_{\mathrm{c}}$, as is the case for all superconductors. In the presence of external fields such as aerogel-induced disorder, the dipole energy and the frequency shift in equation (2) depend on the orientations of $\hat{\mathbf{l}}, \hat{\mathbf{d}}$ and $\mathbf{H}$ (Supplementary Information).

Previous NMR studies of superfluid ${ }^{3} \mathrm{He}$ in aerogel have resulted in a wide range of temperature-dependent frequency shifts accompanied by significant broadening of the NMR line suggestive of a distribution in $\Delta \omega_{\mathrm{A}}$ (refs 13-19). It is likely that macroscopic inhomogeneity and anisotropy in the aerogel influence the orientation of $\hat{\mathbf{l}}$ (Supplementary Information). To avoid this problem, we have grown highly homogeneous isotropic aerogel with a $98.2 \%$ porosity that we have shown to be free of strain with an accuracy of $0.012 \%$ (refs 20,21) using optical birefringence before and after the NMR experiments. The aerogel sample has a cylindrical shape as shown in Fig. 1c, with magnetic field, $\mathbf{H}$, along the vertical $z$ axis. Warming from our lowest temperatures we identified two superfluid aerogel phase ${ }^{21}$, first the B (isotropic)phase and then the A (axial)-phase as shown in Fig. 1d, familiar from pure ${ }^{3} \mathrm{He}$, but with a $25 \%$ suppression of the order parameter amplitude at the pressure $P=26.1$ bar. In the present work, we perform NMR measurements comparing warming and cooling following the horizontal black dashed line in Fig. 1d through all of the superfluid transitions at $P=26.3$ bar.

The phase transitions from $\mathrm{B}$ to $\mathrm{A}$ on warming, and $\mathrm{A}$ to $\mathrm{B}$ on cooling, are identified by a discontinuity in nuclear magnetic susceptibility, $\chi_{\mathrm{A}(\mathrm{B})}$, characteristic of a first order thermodynamic transition (Fig. 2a). The transitions are very sharp on both warming and cooling, with transition width $\approx 0.2 \% T_{\mathrm{c}}$, indicative of a highly homogeneous sample ${ }^{21}$, with typical NMR spectra shown in Fig. 2b. In Fig. 2c we provide an explicit demonstration that the A-phase obtained on warming is indeed the axial superfluid state by measuring its frequency shift as a function of the NMR tip angle, $\beta$ (Methods), and comparing with theory ${ }^{22}$.

The warming transitions shown in Fig. 3 have quadratic field dependence, as expected from Ginzburg-Landau theory ${ }^{23,24}$, precisely mimicked by cooling transitions that supercool by 

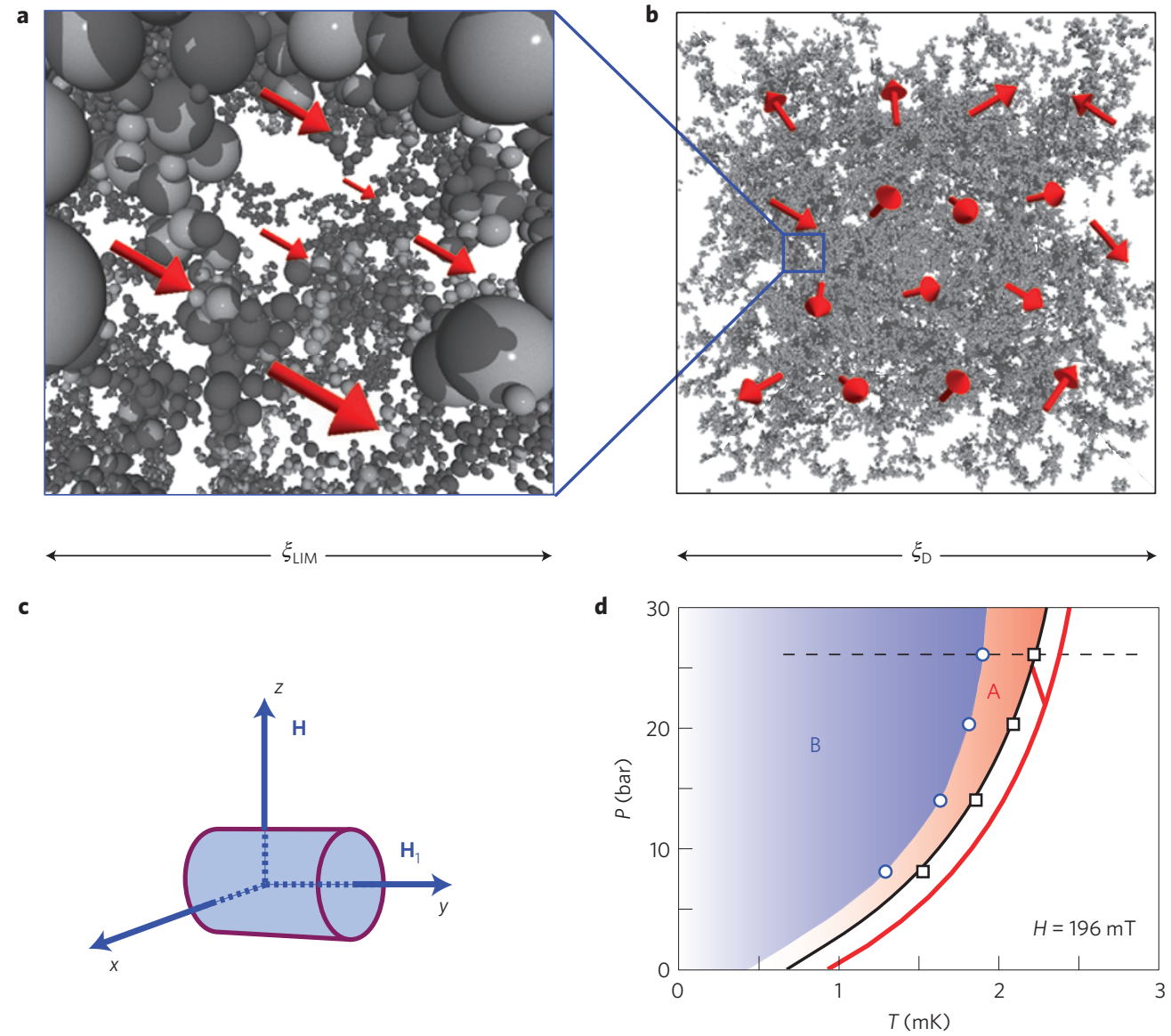

Figure 1 | Superfluid ${ }^{3} \mathrm{He}$ in aerogel. a,b, Numerical simulation of a $98 \%$ aeroge ${ }^{29}$ using a diffusion-limited cluster aggregation algorithm. Red arrows represent the orbital angular momentum distribution for a LIM state, uniform on a short length scale, $\xi_{\mathrm{LIM}}$, called the LIM length, but disordered on a length scale smaller than the dipole length, $\xi_{\mathrm{D}} \sim 8 \mu \mathrm{m}$. c, Geometry of the experimental set-up. The cylinder axis of the aerogel sample and the radiofrequency field $\mathbf{H}_{1}$ are both along the $y$ axis whereas the external magnetic field, $\mathbf{H}$, is oriented along the vertical $z$ axis. $\mathbf{d}$, Pressure versus temperature phase diagram for superfluid ${ }^{3} \mathrm{He}$ for $\mathrm{H}=196 \mathrm{mT}$ imbibed in an isotropic aeroge ${ }^{21}$, the same as for the present work. The open squares are the superfluid phase transitions and the open circles are the B- to A-phase transitions on warming. The solid red curves in the background correspond to $T_{\mathrm{C}}$ and $T_{\mathrm{BA}}$ for pure ${ }^{3} \mathrm{He}$ in zero magnetic field.
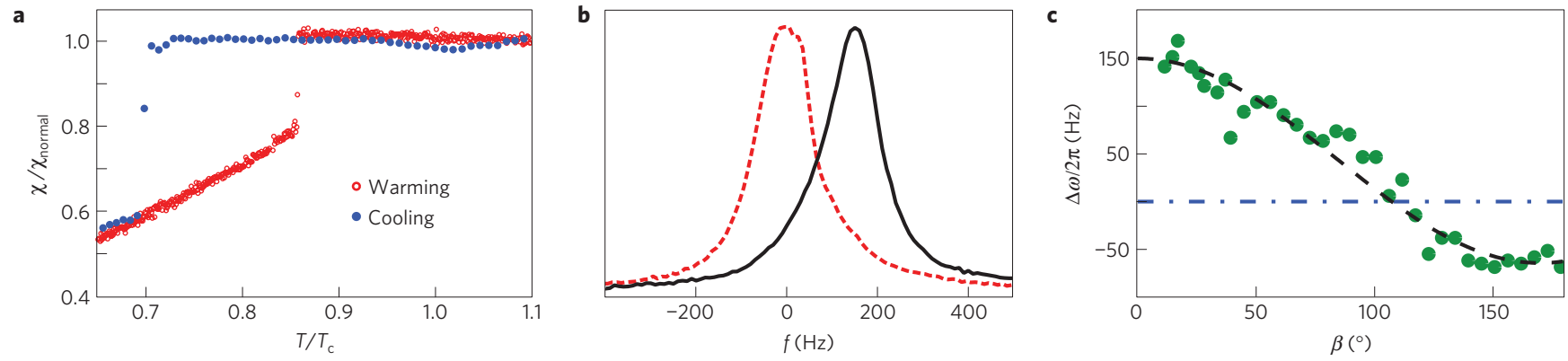

Figure 2 | Identification of the superfluid state. a, Liquid susceptibility relative to the normal state on warming (open red circles) and cooling (filled blue circles) versus reduced temperature in $H=196 \mathrm{mT}$. The data are spaced at $\approx 2 \mu \mathrm{K}$ intervals on warming and $\approx 15 \mu \mathrm{K}$ on cooling. The liquid susceptibility was obtained after subtracting the contribution from the several layers of solid ${ }^{3} \mathrm{He}$ adsorbed to the surface of aerogel, well known to have a Curie-Weiss temperature dependence ${ }^{13,21,30}$. The jump in susceptibility on cooling or warming marks first-order transitions, $T_{\mathrm{AB}}\left(T_{\mathrm{BA}}\right)$. The transition temperature $T_{\mathrm{C}}$ is determined as the point of onset of NMR frequency shifts. b, NMR spectra for ${ }^{3} \mathrm{He}$ in aerogel in the normal state (red dashed curve) and the A-phase (black curve), obtained in $H=196 \mathrm{mT}$ and $P=26.3$ bar on warming from the B-phase at $T / T_{\mathrm{c}}=0.85$. c. Frequency shift versus NMR tip angle $\beta$ for the A-phase nucleated from the B-phase in $H=196 \mathrm{mT}$ (green circles). The dash-dot blue line is the bare Larmor frequency, $\omega_{\mathrm{L}}$, and the dashed black curve is the fit to the theory ${ }^{22}$ for the dipole ordered axial state.

$\sim 300 \mu \mathrm{K}$, an interval that is independent of magnetic field. Extrapolation of the warming transition, $T_{\mathrm{BA}}(H)$, to zero field gives $T_{\mathrm{BA}}(0)-T_{\mathrm{c}}=0.8 \pm 16.3 \mu \mathrm{K}$, indicating that for $H=0$, the equilibrium state is the isotropic B-phase.
In Fig. 4a we show our measurements of the temperature dependence of the frequency shift of the A-phase, $\Delta \omega_{\mathrm{A}}$ (equation (2)), on cooling from the normal state (blue circles) compared with warming from the B-phase (red circles). This history dependence 


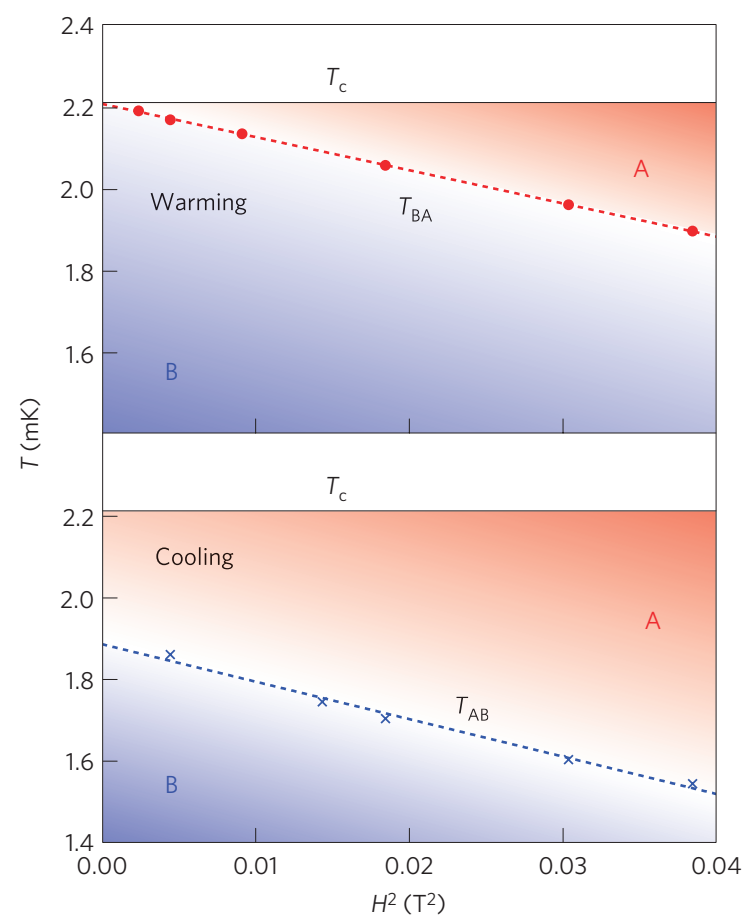

Figure 3 | Superfluid phase diagrams. Superfluid phase diagram $T\left(H^{2}\right)$ for ${ }^{3} \mathrm{He}$ at a pressure of $P=26.3$ bar, for warming from the B-phase (upper panel) contrasted with cooling from the normal fluid (lower panel). The solid black line is $T_{\mathrm{C}}=2.213 \mathrm{mK}$, independent of the magnetic field. The red circles are $T_{\mathrm{BA}}$ corresponding to $\mathrm{B}$ - to $\mathrm{A}$-phase transitions on warming; the blue crosses are $T_{\mathrm{AB}}$, the $\mathrm{A}$ - to $\mathrm{B}$-phase transition after supercooling uniformly by $\approx 300 \mu \mathrm{K}$. The precise parallel behaviour of $T_{\mathrm{AB}}\left(H^{2}\right)$ and $T_{\mathrm{BA}}\left(H^{2}\right)$ indicates that the two phases, formed by warming from the B-phase and by cooling from the normal fluid, are the same state.

is unprecedented. At first glance it might seem that our observation of exactly zero shift on cooling corresponds to a strongly inhomogeneous distribution of frequency shifts that average to zero. However, the NMR line shape is identical to the normal state, evident from the linewidth in Fig. 4b, ruling out an inhomogeneous distribution of shifts. Rather, we infer that cooling from the normal state generates a disordered, but spatially homogeneous, superfluid order parameter in the predicted LIM state ${ }^{25}$. In contrast, the A-phase produced by warming from the B-phase (Fig. 2b) has a uniform frequency shift corresponding to an axial state with the minimum possible dipole energy ${ }^{21}$ shown by the black dashed curve in Fig. 4a. As in the case of pure ${ }^{3} \mathrm{He}$, this behaviour is a manifestation of long-range orientational order. A possible reason for maximal order in the warming experiment can be associated with the presence of a phase boundary at this first-order transition, orienting the angular momentum ${ }^{12,26}$ and breaking rotational symmetry during the formation of the A-phase, thereby inhibiting a LIM state. Another possibility is that the LIM state is disfavoured at low temperatures, an explanation that will require theoretical justification. In addition, it is worth noting that the superfluid transition from the normal state in aerogel is a second-order thermodynamic transition and has no known hysteresis on cooling compared with warming ${ }^{27}$.

The direction of $\hat{\mathbf{I}}$ for ${ }^{3} \mathrm{He}$ in the presence of aerogel should be locally uniform at least over a sufficiently small length scale $\xi_{\text {LIM }}$ (Fig. 1a) that depends on the degree of disorder; Volovik estimated this to be $\sim 1 \mu \mathrm{m}$ (ref. 25 and Supplementary Information). The direction of $\hat{\mathbf{d}}$ must be uniform over a distance $\xi_{\mathrm{D}} \sim 8 \mu \mathrm{m}$ (Fig. $\left.1 \mathrm{~b}\right)^{28}$, called a dipole length determined by the balance between dipole energy and gradient energy. In the limit that $\xi_{\mathrm{LIM}}<\xi_{\mathrm{D}}$, $\hat{\mathbf{l}}$ is randomly oriented with respect to $\hat{\mathbf{d}}$. As a result, the frequency

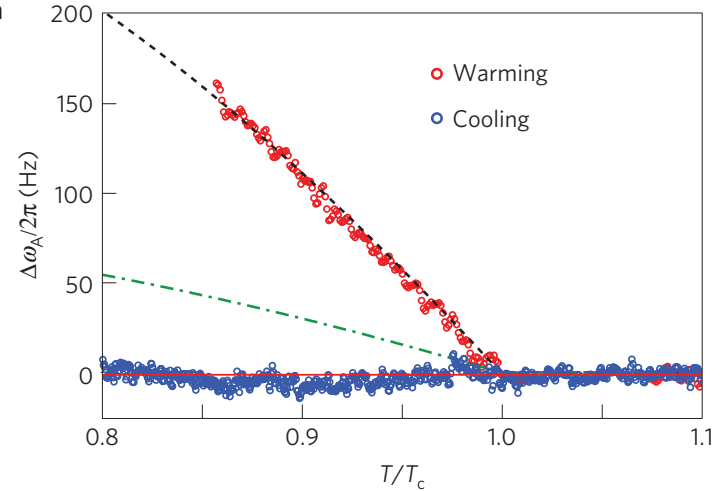

b

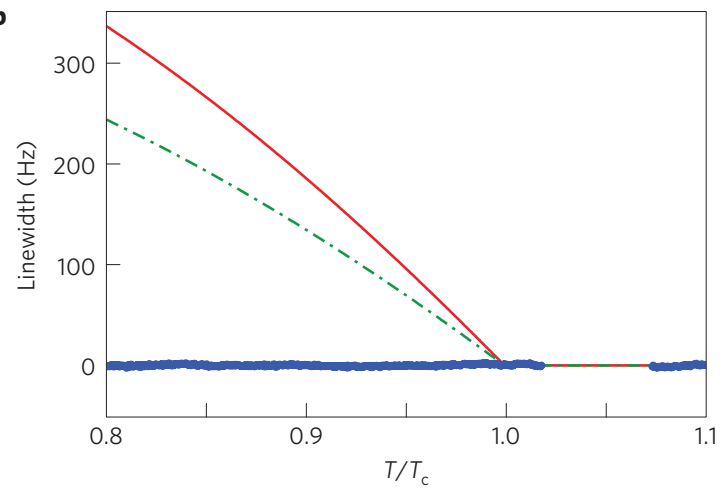

Figure 4 | Identification of the glass phase. Comparison of spectra for cooling and warming in $H=196 \mathrm{mT}$ with small NMR tip angle $\beta=8^{\circ}$ versus reduced temperature. a, Frequency shift on cooling (blue circles) from the normal state compared with warming (red circles) from the B-phase. The black dashed line is the frequency shift of the dipole-ordered axial state having an order parameter suppression of $25 \%$. This suppression was inferred from the frequency shift measurements in the B-phase ${ }^{21}$ and the theoretically defined ratio of the shift of the isotropic state to the axial state for a dipole-ordered configuration, $\hat{\mathbf{I}} \| \hat{\mathbf{d}}$, given by the Leggett relation ${ }^{12}$ and equation (2), $\Omega_{\mathrm{B}}^{2} / \Omega_{\mathrm{A}}^{2}=(5 / 2)\left(\chi_{\mathrm{A}} \Delta_{\mathrm{B}}^{2} / \chi_{\mathrm{B}} \Delta_{\mathrm{A}}^{2}\right)$. The red solid (green dash-dot) curve corresponds to the average frequency shift of a random 2D-disordered (3D-disordered) state. b. The additional contribution to the NMR linewidth beyond that of the normal state measured on cooling (blue circles). The linewidth data for warming are the same (Fig. 2b) but are omitted for clarity. The red solid (green dash-dot) curve is the linewidth of a simulated random 2D-disordered (3D-disordered) state.

shift collapses to zero from its equilibrium value expressed by equation (2) and the linewidth of the NMR spectrum will be identical to that of the normal state $\mathrm{e}^{25}$ (Supplementary Information). In the other limit, $\xi_{\text {LIM }}>\xi_{\mathrm{D}}$, $\hat{\mathbf{d}}$ can follow the projection of $\hat{\mathbf{l}}$ onto the plane perpendicular to $\mathbf{H}$. Then the NMR spectrum will be inhomogeneously broadened and, in general, the average frequency shift will be non-zero. It has been established ${ }^{17}$ that macroscopic strain in aerogel orients $\hat{\mathbf{I}}$ over length scales larger than $\xi_{\mathrm{D}}$, so that inhomogeneity or anisotropy in partially strained samples ${ }^{18,19}$ might obscure the LIM effect.

In the LIM state, the orbital angular momentum has no preferred direction down to and below the $10 \mu \mathrm{m}$ scale given by the dipole length, $\xi_{\mathrm{D}}$, that is, $\xi_{\mathrm{LIM}}<\xi_{\mathrm{D}}$. As such it is very different from a macroscopically inhomogeneous state induced by non-uniformity in density or anisotropy in the aerogel. To illustrate the latter, we consider two models: the 2D-disordered state where the orbital angular momentum is randomly disordered in a plane, for example, the $x z$ or $y z$-plane (Fig. 1c); and the 3Ddisordered state where the orbital angular momentum is random in three dimensions. 


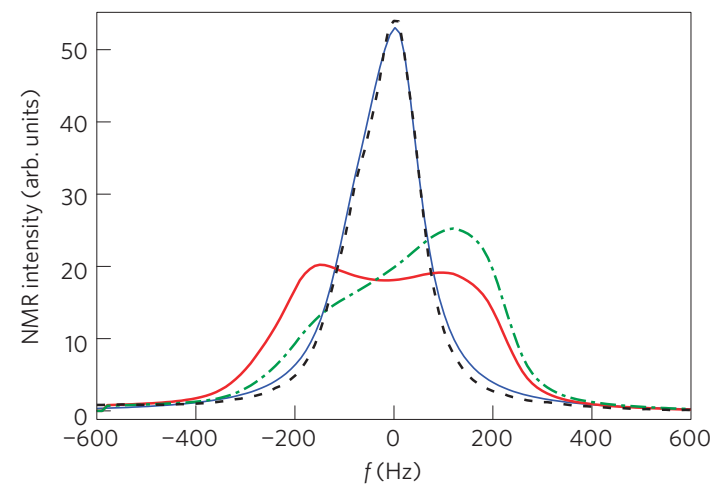

Figure 5 | Comparing the glass phase with macroscopic inhomogeneity. Simulated NMR spectra for 2D-disordered and 3D-disordered distributions of I compared with measured spectra in the normal state (dashed curve) and in the A-phase on cooling (blue curve) in $H=196 \mathrm{mT}$ at a temperature of $T / T_{\mathrm{C}}=0.85$. A LIM state spectrum should have no frequency shift nor linewidth broadening compared to the normal state spectrum, as is evident in our measured spectra. However, the simulated 2D-disordered spectrum (red solid curve) and the 3D-disordered spectrum (green dash-dot curve) are significantly different, notably with an increase in linewidth in both cases and an increase in shift for 3D disorder.

To compare our measurements with possible macroscopic distributions of angular momentum we have simulated the NMR spectra for these two models by calculating the convolution of the normal-state spectrum with spectra corresponding to the probability distribution for the orientation of the order parameter in each model. The result in Fig. 5 for the 3D-disordered state is the green dash-dot curve; the red solid curve is for $2 \mathrm{D}$ disorder. We obtained the frequency shift from the first moment of the simulated NMR spectrum and we calculated the linewidth from the second moment (Supplementary Information). In both cases, we compared results with our measurements in Fig. 4.

The 3D-disordered state has a positive shift that deviates from the data for both warming and cooling experiments (green dashdot curve, Fig. 4). However, the 2D-disordered state (red solid curve) has zero frequency shift, indistinguishable from our cooling measurements and that of the LIM state. On the basis of frequency shift alone it is impossible to identify a LIM superfluid glass as distinct from a 2D macroscopically disordered distribution of the angular momentum. On the other hand, the linewidths for both the $2 \mathrm{D}$-disordered and the $3 \mathrm{D}$-disordered states have significant increases according to our simulation as compared with a LIM state. Our observations are inconsistent with models having significant macroscopic inhomogeneity, supporting the conclusion that we have observed the 3D-LIM effect for superfluid ${ }^{3} \mathrm{He}-\mathrm{A}$ when it is cooled from the normal state.

In earlier NMR work on ${ }^{3} \mathrm{He}$ in anisotropic aerogels, Elbs et al. ${ }^{18}$ and Dmitriev et al. ${ }^{19}$ report evidence for a 2D-LIM effect. The spectra of ref. 19 for three different samples have some similarity to our model calculation for a 3D macroscopically disordered state; that is, they exhibit positive frequency shifts of various amounts for three different samples and significant linewidth broadening compared with the normal state. Nonetheless, Dmitriev et al. performed NMR tip angle and magnetic field orientation experiments and reported that these are consistent with a 2D-LIM state, indicating that there is a LIM effect present, but superposed with effects of macroscopic inhomogeneity in the aerogel.

Finally, we note that in the absence of our ability to switch off the LIM effect, we would not have been able to detect the presence of a superfluid until it appeared suddenly on cooling as a very unusual first-order transition to the B-phase. For sufficiently large magnetic fields such as to suppress the B-phase, evidence from NMR spectra for superfluid ${ }^{3} \mathrm{He}$ would be completely hidden.

\section{Methods}

The aerogel sample is the same as we used previously ${ }^{21}$, having a cylindrical shape (Fig. 1c), $4.0 \mathrm{~mm}$ in diameter and $5.1 \mathrm{~mm}$ long, with a measured porosity of $98.2 \%$. It was grown using the one-step sol-gel method ${ }^{20}$ and characterized thoroughly with both optical-birefringence, cross-polarization techniques and, on similarly prepared samples, small-angle X-ray scattering ${ }^{20,21}$. We found the aerogel to be uniformly isotropic with resolution better than $20 \mu \mathrm{m}^{2}$. We performed pulsed NMR experiments at a pressure $P=26.3$ bar in a magnetic field range from $H=49.9$ to $196 \mathrm{mT}$. The $H_{1}$ field that generates the radiofrequency pulse was oriented parallel to the cylindrical axis. The radiofrequency pulse tips the nuclear magnetization by an angle $\beta$ away from the external field. A Fourier transform of the free induction decay signal of the magnetization in the time domain was phase corrected to obtain the absorption spectrum. The magnetic susceptibility, $\chi$, was determined from the numerical integral of the phase-corrected absorption spectrum. Linewidths were calculated from the square-root of the relative second moment of the spectrum. The sample was cooled by adiabatic demagnetization of $\mathrm{PrNi}_{5}$ to a minimum temperature of $650 \mu \mathrm{K}$ and NMR measurements with a constant small tip angle $\beta$ were performed while the sample warmed or cooled slowly through all of the superfluid transitions, at varying rates of $\sim 3-10 \mu \mathrm{K} \mathrm{h}^{-1}$. Thermometry was based on ${ }^{195} \mathrm{Pt}$ NMR calibrated relative to the known transition temperatures of pure ${ }^{3} \mathrm{He}$.

Received 4 June 2013; accepted 4 October 2013; published online 24 November 2013

\section{References}

1. Larkin, A. I. Effect of inhomogeneities on the structure of the mixed state of superconductors. JETP Lett. 31, 784-786 (1970).

2. Imry, Y. \& Ma, S. Random-field instability of the ordered state of continuous symmetry. Phys. Rev. Lett. 35, 1399-1401 (1975).

3. Gingras, M. J. P. \& Huse, D. A. Topological defects in the random-field XY model and the pinned vortex lattice to vortex glass transition in type-II superconductors. Phys. Rev. B 53, 15193-15200 (1996).

4. Emig, T., Bogner, S. \& Nattermann, T. Nonuniversal quasi-long-range order in the glassy phase of impure superconductors. Phys. Rev. Lett. 83, 400-403 (1999).

5. Simon, J. et al. Quantum simulation of antiferromagnetic spin chains in an optical lattice. Nature 472, 307-312 (2011).

6. Fisher, D. S., Doussal, P. L. \& Monthus, P. Nonequilibrium dynamics of random field Ising spin chains: Exact results via real space renormalization group. Phys. Rev. E 64, 066107 (2001).

7. Radzihovsky, L. \& Toner, J. Smectic liquid crystals in random environments. Phys. Rev. B 60, 206-257 (1999).

8. Bellini, T. et al. Nematics with quenched disorder: What is left when long range order is disrupted? Phys. Rev. Lett. 85, 1008-1011 (2000).

9. Volovik, G. E. Glass state of superfluid ${ }^{3} \mathrm{He}-\mathrm{A}$ in an aerogel. JETP Lett. 63, 301-304 (1996).

10. Norman, M. R. Unconventional superconductivity. http://arxiv.org/abs/1302.3176 (2013).

11. Mydosh, J. A. \& Oppeneer, P. M. Colloquium: Hidden order, superconductivity, and magnetism: The unsolved case of $\mathrm{URu}_{2} \mathrm{Si}_{2}$. Rev. Mod. Phys. 83, 1301-1322 (2011).

12. Leggett, A. J. A theoretical description of the new phases of liquid ${ }^{3} \mathrm{He}$. Rev. Mod. Phys. 47, 331-414 (1975).

13. Sprague, D. T. et al. Homogeneous equal-spin pairing superfluid state of ${ }^{3} \mathrm{He}$ in aerogel. Phys. Rev. Lett. 75, 661-664 (1995).

14. Barker, B. I., Lee, Y., Polukhina, L. \& Osheroff, D. D. Observation of a superfluid ${ }^{3} \mathrm{He}$ A-B phase transition in silica aerogel. Phys. Rev. Lett. 85, 2148-2151 (2000).

15. Baumgardner, J. E. \& Osheroff, D. D. Phase diagram of superfluid ${ }^{3} \mathrm{He}$ in 99.3\% porosity aerogel. Phys. Rev. Lett. 93, 155301 (2004).

16. Nakagawa, H. et al. Equal-spin-pairing superfluid phase of ${ }^{3} \mathrm{He}$ in an aerogel acting as an impurity. Phys. Rev. B 76, 172504 (2007).

17. Kunimatsu, T. et al. Orientation effect on superfluid ${ }^{3} \mathrm{He}$ in anisotropic aerogel. JETP Lett. 86, 216-220 (2007).

18. Elbs, J., Bunkov, Y. M., Collin, E. \& Godfrin, H. Strong orientational effect of stretched aerogel on the ${ }^{3} \mathrm{He}$ order parameter. Phys. Rev. Lett. 100, 215304 (2008).

19. Dmitriev, V. V. et al. Orbital glass and spin glass states of ${ }^{3} \mathrm{He}-\mathrm{A}$ in aerogel. JETP Lett. 91, 599-606 (2010).

20. Pollanen, J. et al. Globally anisotropic high porosity silica aerogels. J. Non-Cryst. Solids 354, 4668-4674 (2008).

21. Pollanen, J., Li, J. I. A., Collett, C. A., Gannon, W. J. \& Halperin, W. P. Identification of superfluid phases of ${ }^{3} \mathrm{He}$ in uniformly isotropic $98.2 \%$ aerogel. Phys. Rev. Lett. 107, 195301 (2011). 
22. Brinkman, W. F. \& Smith, H. Frequency shifts in pulsed NMR for ${ }^{3} \mathrm{He}(\mathrm{A})$. Phys. Lett. 51, 449-450 (1975).

23. Thuneberg, E. V., Yip, S. K., Fogelström, M. \& Sauls, J. A. Models for superfluid ${ }^{3}$ He in aerogel. Phys. Rev. Lett. 80, 2861-2864 (1998).

24. Halperin, W. P., Choi, H., Davis, J. P. \& Pollanen, J. Impurity effects of aerogel in superfluid ${ }^{3}$ He. J. Phys. Soc. Jpn 77, 111002 (2008).

25. Volovik, G. E. On Larkin-Imry-Ma state of ${ }^{3} \mathrm{He}-\mathrm{A}$ in aerogel. J. Low Temp. Phys. 150, 453-463 (2008).

26. Bradley, D. I. et al. Relic topological defects from brane annihilation simulated in superfluid ${ }^{3}$ He. Nature Phys. 4, 46-49 (2008).

27. Bennett, R. G. et al. Modification of the ${ }^{3} \mathrm{He}$ phase diagram by anisotropic disorder. Phys. Rev. Lett. 107, 235504 (2011).

28. Vollhardt, D. \& Wölfle, P. The Superfluid Phases of Helium 3 (Taylor and Francis, 1990).

29. Pollanen, J. et al. New chiral phases of superfluid ${ }^{3} \mathrm{He}$ stabilized by anisotropic silica aerogel. Nature Phys. 8, 317-320 (2012).

30. Bradley, D. I. et al. Magnetic phase transition in a nanonetwork of solid ${ }^{3} \mathrm{He}$ in aerogel. Phys. Rev. Lett. 105, 125303 (2010).

\section{Acknowledgements}

We are grateful to V. V. Dmitriev, M. R. Eskildsen, M. J. P. Gingras, R. Ikeda, J. A. Sauls, J. Saunders, D. Vollhardt and G. E. Volovik for helpful comments and for support from the National Science Foundation, DMR-1103625.

\section{Author contributions}

The experiment is designed by J.I.A.L., J.P. and W.P.H. Experimental work and analysis was principally carried out by J.I.A.L. assisted by J.P. and A.M.Z. with further support from C.A.C. and W.J.G. Advice and assistance were provided by W.P.H.

\section{Additional information}

Supplementary information is available in the online version of the paper. Reprints and permissions information is available online at www.nature.com/reprints. Correspondence and requests for materials should be addressed to W.P.H.

\section{Competing financial interests}

The authors declare no competing financial interests. 